\title{
Soluzioni generalizzate e disuguaglianze variazionali per alcuni sistemi differenziali non lineari della fisica matematica $(*)$.
}

\author{
Mauro Fabrizio (Bologna) (**)
}

Sunto. - Si ricercano le condizioni di correttezza per sistemi differenziali del primo ordine ai tipo iperbolico. Tali condizioni risultano legate a restrizioni fisiche, in particolare termodinamiche. Pertanto dopo uno studio della termodinamica dei processi retti allle equazioni considerate si perviene ad una definizione di soluzione generalizzata, esprimibile mediante una disuguaglianza variazionale. In tale nuovo formulazione è contenuto sia il sistema delle equazioni differenziali che descrivono il processo fisico e sia una limitazione di natura termodinamica. Per il problema di Cauchy relativo ai sistemi in esame si stabilisce poi nel campo delle soluzioni generalizzate una disuguaglianza integrale sulla energia interna e quindi sotto opportune ipotesi un teorema di unicita.

\section{Introduzione.}

In questo studio considereremo una classe di processi fisici che variano col tempo e che sono governati da sistemi di equazioni differenziali del primo ordine:

$$
u_{t}+A(u) u_{x}=0 \quad\left(u_{t}=\frac{\partial u}{\partial t}, u_{x}=\frac{\partial u}{\partial x}\right)
$$

dove $u=\left(u_{1}, u_{2}, \ldots, u_{n}\right)$ è una $n$-upla di funzioni scalari di $x, t$ con $x \in(-\infty, \infty)$, $t \in[0, T](T>0)$ e $A$ è una matrice $n \times n$ che per ogni $u \in(-\infty, \infty)$ ha autovalori reali e distinti. Il sistema (1) risulta quindi iperbolico secondo la definizione di Petrovskii.

Per tali sistemi studieremo il problema di Cauchy. Cercheremo cioè una soluzione $u(x, t)$ di $(1)$ tale che:

$$
\left.u\right|_{t=0}=u^{0}(x)
$$

essendo $u^{0}(x)$ una funzione nota e definita in $(-\infty, \infty)$.

La propagazione ondosa unidimensionale relativa ai fenomeni di gasdinamica di elasticità non lineare, di magnetofluidodinamica, di ottica non lineare [1], [2], [3] ̀̀ descritta da sistemi di equazioni del tipo (1).

Infatti ad esempio il sistema delle equazioni della gasdinamica relative ad un processo adiabatico unidimensionale è esprimibile, mediante le variabili euleriane,

(*) Lavoro eseguito nell'ambito dei gruppi di ricerca per la Matematica del C.N.R.

(**) Entrato in Redazione il 31 dicembre 1971. 
dalle equazioni:

$$
\begin{aligned}
& \frac{\partial \varrho}{\partial t}+\frac{\partial}{\partial x}(\varrho v)=0 \\
& \frac{\partial(\varrho v)}{\partial t}+\frac{\partial}{\partial x}\left(p+\varrho v^{2}\right)=0
\end{aligned}
$$

dove $\varrho$ è la densità, $v$ la velocità, $p$ la pressione. Passando a nuove variabili (lagrangiane) è possibile scrivere tale sistema nella forma:

$$
\begin{aligned}
& \frac{\partial \mu}{\partial t}-\frac{\partial v}{\partial x}=0 \\
& \frac{\partial v}{\partial t}+\frac{\partial p}{\partial x}=0
\end{aligned}
$$

dove $\mu=1 / \varrho$. Formajmente tale trasformazione può essere ottenuta per mezzo delle formule:

$$
\frac{\partial}{\partial x}=\varrho \frac{\partial}{\partial x^{\prime}} ; \frac{\partial}{\partial t}=\frac{\partial}{\partial t^{\prime}}-\varrho v \frac{\partial}{\partial x^{\prime}}
$$

dove, per evitare equivoci, abbiamo indicato con $x, t$ e $x^{\prime}, t^{\prime}$ rispettivamente le variabili euleriane e quelle lagrangiane.

E facile verificare mediante semplici esempi [4] che il problema di Cauchy (1), (2) non è corretto in quanto non sussistono per un intervallo di tempo arbitrario teoremi di stabilità ; mentre, solo in casi molto particolari, si conoscono teoremi di esistenza [3]. Inoltre è interessante osservare che l'unicità non sussiste se non si impongono, oltre ad alcune restrizioni sulla $A(u)$, anche condizioni sull'aumento dell'entropia sulle discontinuità delle soluzioni. Tale condizione è conseguenza del fatto che i sistemi (1) descrivono in generale processi irreversibili nel tempo [2], [3].

Queste osservazioni di notevole importanza fanno capire come i fenomeni fisici considerati non possono essere compiutamente descritti soltanto dalle equazioni di moto (1), quindi per pervenire ad una formulazione corretta del problema (1), (2) occorre aggiungere ulteriori restrizioni che consentono di descrivere compiutamente i processi fisici considerati. A tale proposito è noto, che il secondo principio della termodinamica svolge un ruolo determinante al fine di stabilire tali condizioni di correttezza, poichè caratterizza in modo opportuno l'insieme delle matrici $A(u)$ e quello delle soluzioni $u$.

In questa nota pertanto, mediante una definizione generalizzata di soluzione che deriva appunto da considerazioni termodinamiche ed esprimibile mediante, una disuguaglianza variazionale, stabilisco nella classe delle funzioni limitate e misurabili una disuguaglianza integrale sull'energia interna che costituisce un contributo interessante per il teorema di unicità. Infatti tale risultato mi ha portato a formulare, una limitazione nel cui campo di validità è possibile pervenire ad un teorema di unicità.

È opportuno osservare che recentemente J. LERAY e Y. OHYA [5] hanno affrontato, facendo uso di un algoritmo matematico alquanto raffinato e potente, lo studio di sistemi iperbolici quasi-lineari del tipo (1). 
MaURo FABRIzIo: Soluzioni generalizzate e disuguaglianze variazionali, ece.

Essi pervengono, per il problema di Cauchy, soltanto a teoremi di unicità e stabilità in piccolo, cioè per un intervallo di tempo sufficientemente piccolo. Questa limitazione, nella impostazione del lavoro [5] ci sembra non eliminabile ed è coerente col fatto che in [5] non si libera la classe delle soluzioni di (1) da quelle non soddisfacenti il secondo principio della termodinamica.

1. - Poichè siamo interessati ad equazioni che descrivono processi fisici, considereremo soltanto sistemi di equazioni di tipo conservativo

$$
u_{t}+E f_{x}(u)=0
$$

dove $E$ è una matrice $n \times n$ simmetrica e costante, $f=\left(f_{1}, f_{2}, \ldots, f_{n}\right)$ è una $n$-upla di funzioni scalari di $u$ che supporemo sufficientemente regolare.

Le equazioni della gasdinamica, della elasticità non lineare, della magnetofluidodinamica, dell'ottica non lineare [3] sono effettivamente del tipo (5). Se poniamo infatti, sempre nel caso della gasdinamica:

$$
E=\left\|\begin{array}{ll}
0 & 1 \\
1 & 0
\end{array}\right\| \quad u=\left\|\begin{array}{l}
\mu \\
v
\end{array}\right\| \quad f=\left\|\begin{array}{r}
p \\
-v
\end{array}\right\|
$$

possiamo esprimere il sistema (3), (4) mediante l'espressione (5).

Valendoci sempre del secondo principio della termodinamica dimostreremo che i sistemi considerati, espressi dalla equazione (5) possiamo classificarli fra i sistemi della forma:

$$
u_{\imath}+E \mathfrak{Q}(u) u_{x}=0
$$

dove $\mathfrak{U}(u)$ è una matrice simmetrica definita negativa. Generalizzando il risultato di FrIEdrichs [5] e J. Leray si verifica che codesti sistemi sono una particolare classe di sistemi iperbolici, eioè quelli che ammettono un integrale dell'energia.

Inoltre è importante associare a (5) anche il sistema:

$$
u_{t}+E f_{x}(u)=\left(B u_{x}\right)_{x}
$$

dove $B$ è anch'essa una matrice $n \times n$ simmetrica definita 0 semidefinita positiva che può dipendere da $u$. Questo sistema di equazioni descrive ancora la propagazione ondosa relativa a fenomeni di gasdinamica, elasticità non lineare, ecc. quando però il mezzo è dissipativo. Il termine a secondo membro infatti interviene per descrivere fenomeni di dissipazione come sforzi viscosi, conduzione di calore, ecc.

Infine ricordiamo che per il sistema (5) è possibile dare una definizione debote di soluzione.

DefiNIzIONe 1. - Una funzione $u(x, t)$ limitata e misurabile è soluzione debole del sistema (5) in $Q=(-\infty, \infty) \times[0, T]$ se per ogni funzione $\Psi \in C^{\mathrm{I}}(Q)$ a valori in $R^{n}$

5 - Annali di Matematira 
e a supporto compatto in $(-\infty, \infty) \times(0, T)$ :

$$
\int_{Q}\left[\left(u, \Psi_{t}\right)+\left(E f(u), \Psi_{s}\right)\right] d x d t=0
$$

dove con $(\cdot, \cdot)$ indichiamo il prodotto scalare in $R^{n}$.

Ricordiamo che ogni soluzione regolare di (5) è anche soluzione debole nel senso della Definizione 1.

2. - Ora brevemente riassumeremo le proprietà delle soluzioni deboli di un sistema di equazioni quasilineari del tipo (5) confrontandole eventualmente con le soluzioni del problema lineare (vedi [2]).

1) Le discontinuità delle soluzioni sono una proprietà caratteristica dei sistemi quasi-lineari e possono apparire anche con dati iniziali regolari.

2) Una soluzione discontinua di un sistema di equazioni lineari che soddisfa le leggi di conservazione è univocamente determinata mediante $i$ dati iniziali, mentre per $i$ sistemi quasi-lineari ulteriori condizioni sulle discontinuità devono essere imposte per garantire l'unicità [3], [4].

3) Le soluzioni dei sistemi lineari di tipo iperbolico descrivono processi fisici reversibili nel tempo, mentre le soluzioni discontinue dei sistemi quasi-lineari descrivono in generale processi irreversibili ([2], pag. 63).

4) La classe delle soluzioni associata ad un dato sistema di equazioni quasi-lineari dipende dalla forma con la quale l'equazione è scritta (*).

5) Le soluzioni deboli di un sistema quasi-lineare non possono essere ottenute, a differenza del caso lineare, come limite di soluzioni regolari.

Come conseguenza di queste considerazioni sembra naturale imporre ulteriori restrizioni alle soluzioni che pretendono di descrivere un processo fisico.

A questo scopo osserviamo che i processi descritti dal sistema (5), come già detto, ignorano meccanismi di dissipazione come sforzi viscosi, conduzione di calore, conduzione elettrica. Ora da un punto di vista fisico sembra più esatto dire che tali effetti dissipativi si trascurano. Pertanto, se consideriamo un numero $v>0$ e l'equazione:

$$
u_{t}+E f_{x}(u)=\nu\left(B u_{x}\right)_{x}
$$

supporremo che:

(*) Cfr. ad esempio GeL'Fand [1], Godonov [7] dove considerando l'equazione $u_{t}+u u_{x}=0$ si verifica che, poichè tale equazione formalmente pù̀ essere scritta $\left(\frac{1}{2} u^{2}\right)_{t}+\left(\frac{1}{3} u^{3}\right)_{x}=0$, le soluzioni deboli dell'una non sono soluzioni deboli per l'altra e viceversa. 
a) le soluzioni deboli del sistema (5) si debbono ottenere come limite, di soluzioni del sistema (7) per $\boldsymbol{\nu} \rightarrow 0$.

Tale ipotesi fatta da LAx [8] nel 1954 è conseguenza di una ricerca dovuta ad HoPF [9] nella quale viene verificata la validità dell'ipotesi a) per l'equazione $u_{t}+u u_{x}=0$.

Vari autori fra cui V. F. D'YACENKo [10] e VVedEnskaya [11] hanno dimostrato che, anche mediante codeste ipotesi, non è possibile formulare un teorema di unicità per il problema (5), (2). Inoltre è facile dimostrare l'esistenza di soluzioni dotate di particolari discontinuità e non stabili rispetto ad una piccola perturbazione [12].

Una considerazione quindi che discende dalle osservazioni finora fatte è che i problemi fisici che consideriamo sono soltanto in parte descritti mediante il sistema (5) e inoltre la condizione $a$ ) non è sufficiente per rendere il problema corretto sia in senso fisico che in senso matematico.

Per superare tali difficoltà è conveniente riferirsi al problema di Cauchy nel caso in cui il sistema (5) si riduce ad una sola equazione.

In tale caso la OLEǏNIK [4] nel 1957, per pervenire all'unicità, impone la condizione di aumento dell'entropia sulle linee di discontinuità delle soluzioni non regotari. Tale ipotesi, che risulta essenziale nei problemi di unicità e estesa successivamente anche nel caso di sistemi di equazioni [13], [11], è naturale conseguenza della nostra osservazione 3) secondo cui i processi retti da equazioni quasi-lineari descrivono in generali fenomeni irreversibili.

In conseguenza della importanza di codeste considerazioni mi è sembrato naturale imporre come seconda ipotesi di partenza che:

b) le soluzioni del sistema (5) devono soddisfare al secondo principio della termodinamica.

Recentemente Hopf [15] sempre nel caso di una singola equazione del tipo:

$$
v_{t}+a_{x}(v)=0
$$

perviene ad una nuova formulazione di soluzione generalizzata, esprimibile mediante la seguente disuguaglianza:

$$
\int_{Q}\left[\Im(v) \varphi_{i}+\widetilde{\jmath}(v) \varphi_{i}\right] d x d t \geqslant 0
$$

dove $\Im(v)$ è una arbitraria funzione convessa, $\widetilde{F}(v)$ una primitiva di $\Im_{v} a_{v}$ e $\varphi$ una funzione sufficientemente regolare e a supporto compatto in $(-\infty, \infty) \times(0, T)$.

Questa relazione è equivalente alla definizione di soluzione generalizzata data da KRUžKov [16] e da VoLPERT [17]. Codeste definizioni sono certamente interessanti in quanto una funzione che la verifica è soluzione debole di (5) (vedi [15] [16]) e soddisfa alla condizione dell'aumento dell'entropia sulle discontinuità. Mi è sembrato opportuno a questo punto, vedere se l'espressione (8) non si possa estendere al caso di sistemi del tipo (5). 
3. - In questo numero pertanto, ci soffermeremo su aleune considerazioni relative alla termodinamica dei fenomeni retti da sistemi del tipo:

$$
u_{t}+E h_{x}(u)=\tilde{\varrho}(x, t)
$$

dove $\tilde{g}$ è una funzione nota in $Q$ e rappresenta le sorgenti del campo, mentre supporremo il mezzo caratterizzato dalla relazione costitutiva $h=h(u)$. Ovviamente il sistema (9) rappresenta il fenomeno deseritto da (5) quando $h(u) \equiv f(u)$ e $\tilde{\varrho} \equiv 0$.

Inoltre indichiamo rispettivamente con $\varepsilon(x, t), \eta(x, t)$ l'energia interna per unità di massa eomprensiva del contributo dovuto alla energia cinetica e la densità di entropia, con $\boldsymbol{q}(x, t), \boldsymbol{r}(x, t)$ il vettore flusso di calore e lo scalare sorgente di calore relative all'unità di massa, infine con $\theta(x, t) \geqslant 0$ la temperatura assoluta.

Supponiamo ora che il materiale sia caratterizzato dalle tre funzioni:

$$
\varepsilon=\varepsilon(u, \eta), \quad \theta=0(u, \eta), \quad \boldsymbol{q}=\boldsymbol{q}(u, \eta) .
$$

Per i fenomeni che considereremo il primo principio della termodinamica, relativo ad un arbitrario dominio spaziale $\Omega$, assume la forma:

$$
\frac{d}{d t} \int_{\Omega} \varepsilon d m=-\int_{\Omega}\left(u_{t}, \tilde{h}\right) d m+\int_{\partial \Omega} \boldsymbol{q} \cdot \boldsymbol{n} d s+\int_{\Omega} r d m
$$

dove $\partial \Omega$ è la frontiera di $\Omega$ e $\boldsymbol{n}$ la relativa normale orientata verso l'interno.

Verifichiamo ora la validità della equazione (10) ancora nel caso particolare della gas-dinamica. In tale caso un generico mezzo è caratterizzato dalla relazione costitutiva $\mathfrak{p}=\mathfrak{p}(u)$ e il sistema (9) assume la forma:

$$
\begin{aligned}
& \frac{\partial \mu}{\partial t}-\frac{\partial v}{\partial x}=0 \\
& \frac{\partial v}{\partial t}+\frac{\partial p}{\partial x}=\mathfrak{f}
\end{aligned}
$$

dove con f rappresentiamo la forza esterna per unità di massa.

La variazione di energia meccanica $W$ nell'unità di tempo relativa al dominio $\Omega$ è:

$$
W^{r}=-\int_{\Omega} \frac{\partial}{\partial x}(\mathfrak{q} v) d m+\int_{\Omega} \tilde{i} v d m
$$

Pertanto il primo prineipio della termodinamica assume la forma:

$$
\frac{d}{d t} \int_{\Omega} \varepsilon d m=-\int_{\Omega} \frac{\partial}{\partial x}(\mathfrak{p} v) d m+\int_{\Omega} f v d m-\int_{\partial \Omega} \boldsymbol{q} \cdot \boldsymbol{n} d s+\int_{\Omega} r d m
$$


da eui mediante le equazioni di moto (11), (12) si ha:

$$
\frac{d}{d t} \int_{\Omega} \varepsilon d m=\int_{\Omega} v_{t} v d m-\int_{\Omega} \mathfrak{p} \mu_{t} d m-\int_{\partial \Omega} \boldsymbol{q} \cdot \boldsymbol{n} d s+\int_{\Omega} r d m .
$$

È verificata pertanto nel caso della gas-dinamica la validità della equazione (10). Per quanto riguarda il secondo principio della termodinamica noi adotteremo la formulazione dovuta a C. TRUESDELe e R. A. ToupIN (vedi [19], capitolo E) ed esprimibile mediante la disuguaglianza di Clausius-Duhem:

$$
\frac{d}{d t} \int_{\Omega} \eta d m \geqslant \int_{\partial \Omega}^{1} \frac{1}{\theta} \boldsymbol{q} \cdot \boldsymbol{n} d s+\int_{\Omega} \frac{r}{\theta} d m
$$

Supponendo i campi sufficientemente regolari è possibile trasformare (14) in una equazione differenziale e sostituire il risultato nella corrispondente espressione differenziale di (16). Da oui ponendo $g=\nabla \theta$ si ottiene la seguente ben nota forma locale della disugraglianza di Clausius-Duhem:

$$
\varepsilon-\eta \theta+\left(u_{t}, h\right)+\frac{1}{\varrho \theta} q \cdot g \leqslant 0
$$

Ora è noto [20] che mediante la disuguaglianza (17) è possibile stabilire le relazioni:

$$
h=-\nabla_{u} \varepsilon, \quad \theta=\frac{\partial \varepsilon}{\partial \eta}
$$

dove $\nabla_{u} \varepsilon$ è il gradiente di $\varepsilon$ rispetto a $u$. In seguito per una arbitraria funzione scalare $H(u, \eta)$ indicheremo con $H_{\eta}=\partial H / \partial \eta, H_{u}=\nabla_{u} H$.

È necessario ora precisare le ipotesi sotto le quali noi opereremo. Supporremo innanzi tutto il mezzo non conduttore $\left({ }^{*}\right)$, cioè $\boldsymbol{q}=0$ e inoltre poichè abbiamo supposto $h=h(u)$ funzione solo di $u$ non considerando la dipendenza in $\eta$, riterremo a causa della (18), che o $\eta$ durante il processo è una funzione costante o l'energia interna ha la forma:

$$
\varepsilon=J(\eta)+I(u)
$$

per cui

$$
\hbar=-I_{u}, \quad \theta=J_{\eta} .
$$

I due casi risultano equivalenti relativamente alle considerazioni $\theta$ ai. risultati che seguiranno, pertanto noi per fissare le idee ci riferiremo nel seguito soltanto

(*) In effetti tale condizione è conseguenza delle equazioni costitutive e della disuguaglianza di Clausius-Duhem (17). 
al secondo. In tal caso si ha, poichè il sistema (5) è un particolare tipo di sistema (9):

$$
f=-\bar{\varepsilon}_{i b}=-\bar{I}_{z}
$$

dove $\bar{\varepsilon}=\bar{J}(\eta)+\bar{I}(u)$ rappresenta l'energia interna relativa al mezzo rappresentato dal sistema di equazioni (5).

È possibile così giustificare la nostra affermazione secondo cui il sistema (5) è di tipo iperbolico. Infatti per la relazione $f=-\bar{I}_{u}$ deve essere

$$
\mathfrak{U}(u) \stackrel{\text { def }}{=} f_{i u_{j}}=-\bar{I}_{u_{i} u_{j}}
$$

che risulta ovviamente una matrice simmetrica e come verificheremo in seguito definita negativa.

4. - Prendiamo in esame ora l'equazione (7) con $B=E$ (tale ipotesi, dovuta soltanto alla necessità đi semplificare $\mathrm{i}$ calcoli, non è essenziale per quanto riguarda il contenuto del procedimento che segue) e osserviamo che, nell'ipotesi di dati iniziali sufficientemente regolari, le soluzioni del problema di Cauchy relative a tale equazione esistono e sono sufficientemente regolari [21].

Consideriamo ora l'identità, valida per campi sufficientemente regolari:

$$
\dot{I}+\left(u_{t}, h\right)=0
$$

ottenuta da (17) ricordando che $\theta=J_{\eta}$ e l'ipotesi $\boldsymbol{q}=0$. Sostituendo ora in (19) al posto di $u_{i}$ il valore ottenuto da (17) si ha:

$$
\dot{I}-\left(E f_{x}, h\right)+\nu\left(u_{x x}, h\right)=0
$$

quindi

$$
\dot{I}-\left(E f_{x}, h\right)+v\left(u_{x}, h\right)_{x}-v\left(u_{x}, h_{u} u_{x}\right)=0 .
$$

È noto dalla fisica l'importanza del potenziale termodinamico $\varepsilon=\varepsilon(\eta, u)$ che si suppone sempre una funzione convessa dei suoi argomenti. Pertanto la matrice $I_{u u}$, che supporremo, sempre ben definita su tutto $R^{n}$, risulterà anch'essa una funzione convessa di u, cioè:

$$
\left(I_{u u} \xi, \xi\right) \geqslant 0
$$

essendo $\xi$ un vettore a $n$-dimensioni.

È conveniente ora esprimere l'equazione (20) mediante una relazione integrale che consenta una definizione debole di tale espressione.

A questo proposito bisogna ammettere:

$$
-\left(E f_{x}, h\right)=F_{x}(u)
$$


dove $F^{\prime}(u)$ è una funzione a valori scalari di $u$ e necessariamente tale che:

$$
F_{u_{h}}=-E_{i j} h_{j} f_{i u_{h}}
$$

(abbiamo sottointeso, come sempre anche in seguito, il simbolo di sommatoria su. gli indici ripetuti).

Pertanto occorre che la forma differenziale:

$$
E_{i j} h_{j} f_{i u_{k}} d u_{k}
$$

sia integrabile. Ciò è senz'altro vero quando:

$$
\left(E_{j i} h_{i} f_{i u_{k}}\right)_{h}=\left(E_{j i} h_{j} f_{i u_{h}}\right)_{u_{k}}
$$

cioè in virtù delle condizioni $h=-I_{u}$ :

$$
\left(E_{j i} I_{u_{j}} f_{i u_{h}}\right)_{u_{k}}=\left(E_{j i} I_{u_{j}} f_{i u_{k}}\right)_{u_{h}} .
$$

Per $n>2$ tale relazione differenziale presenta più equazioni che incognite; quindi, per una generica $f$, non ammette altre soluzioni she quelle per eui:

$$
I_{u_{i}}=\operatorname{cost}
$$

Fortunatamente la funzione $f(u)$, come dimostrato, deriva anch'essa dal potenziale convesso $\bar{I}(u)$, pertanto $(24)$ si esprime:

$$
E_{j i} I_{u_{j} u_{i b}} \bar{I}_{u_{i} u_{h}}=E_{j i} I_{u_{j} u_{h}} \bar{I}_{u_{i} u_{l}} \text {. }
$$

In questo caso esistono senz'altro soluzioni di tale relazione differenziale, ne sono esempio:

$$
I=\alpha_{0} \bar{I}(u)+\sum_{i=1}^{n} \alpha_{i} u_{i}+\alpha_{n+1}
$$

dove $\alpha_{i}$ sono arbitrari coefficienti di eui $\alpha_{0} \geqslant 0$.

Tale possibilità, che come vedremo risulterà essenziale per una generalizzazione di soluzione analoga alla (8), è conseguenza diretta della ipotesi $b$ ) secondo cui le soluzioni di (5) devono soddisfare al secondo principio della termodinamica.

Inoltre l'insieme delle funzioni convesse $I(u)$ che verificano (25) lo indicheremo con D.

Consideriamo ora una funzione a valori sealari $\varphi(x, t) \geqslant 0$ appartenente alla classe $\Phi$ delle funzioni sufficientemente regolare e a supporto compatto in $Q=(-\infty, \infty) \times$ $\times(0, T)$. Ora da $(20)$ moltiplicando per $\varphi$ e integrando per parti in $Q$ si ottiene:

$$
\int_{Q}\left[I(u) \varphi_{t}+F(u) \varphi_{x}\right] d x d t=-v \int_{Q} I(u) \varphi_{x x} d x d t-v \int_{Q}\left(u_{x}, h_{u} u_{x}\right) \varphi d x d t .
$$


dove $F(u)$ è tale che $F_{u i}=-E_{j r} h_{r} f_{j u l}$. Infine se $\nu \rightarrow 0$ si ha che, poichè $\varphi$ è una funzione regolare, $\varphi_{x w}$ risulta limitata in $Q$ come $I(u)$ e quindi il primo termine a secondo membro di (27) tende a zero. Per quanto riguarda invece il secondo termine, poichè non è possibile assicurare la limitatezza di $u_{x}$ per $v \rightarrow 0$, si ha, in virtù della relazione (21), che il limite per $\nu \rightarrow 0$ deve risultare una quantità non negativa. Pertanto da (27) si ha per $v \rightarrow 0$ e per ogni $I(u)$ :

$$
\int_{e}\left[I(u) \varphi_{t}+F(u) \varphi_{x}\right] d x d t \geqslant 0
$$

Si generalizza così il risultato di Hopf [17] al caso di un sistema del tipo (5).

Non è difficile verificare, seguendo [22], che ogni soluzione di (28) è soluzione debole di (5).

Infatti supponiamo che esista una funzione $u$ che non sia soluzione debole nel senso della Definizione 1, ma che verifichi (28) per ogni $I(u)$.

Consideriamo quindi le particolari funzioni $I(u) \equiv \pm u_{s}$ che ovviamente appartengono a $D$.

Eे facile ora verificare che, ponendo nella disuguaglianza (28) prima $I(u)=u_{s}$ e poi $I(u)=-u_{s}$, la funzione $u$ soluzione di $(28)$ deve soddisfare l'uguaglianza:

$$
\int_{Q}\left(u_{s} \varphi_{t}+E_{s j} f_{j} \varphi_{x}\right) d x d t=0 .
$$

Quindi ogni soluzione della disuguaglianza (28) deve, contrariamente alla nostra ipotesi, necessariamente essere anche soluzione debole del sistema (5) nel senso della Definizione 1.

Da qui pertanto, si vede l'importante contributo di sintesi rappresentato dalla disuguaglianza (28), in quanto in tale formulazione è contenuto sia il sistema delle equazioni di moto e sia una limitazione di natura termodinamica che traduce la legge della perdita dell'energia interna per un gas mantenuto a temperatura o ad entropia costante.

Questo ei consente di pervenire alla seguente definizione generalizzata di soluzione per il problema (5), (2):

DEFINIZIONE 2. - Una funzione $u(x, t)$ limitata e misurabile è una soluzione generalizzata del problema di Cauchy associato al sistema (5) se, nel dominio $Q$ :

1) verifica la disuguaglianza (28) per ogni $\varphi \geqslant 0$ appartenente a $\Phi$ e per ogni funzione convessa $I(u) \in \mathfrak{D}$.

2) per quasi tutti $i t \in[0, T]$ la funzione $u(x, t)$ è tale che per ogni $R \geqslant 0$ :

$$
\lim _{t \rightarrow 0} \int_{|x| \leqslant k}\left|u(x, t)-u^{0}(x)\right| d x=0 .
$$


MAURo FABRIzIo: Soluzioni generalizzate e disuguaglianze variazionali, ecc.

Per codesta soluzione generalizzata è possibile pervenire al seguente teorema:

TEorema - Se la funzione limitata $u(x, t)(|u|<M)$ è soluzione goneralizzata del probtema (5), (2) secondo la DeFinizione 2 , allora risulta por quasi tutti $i t \in[0, T]$ :

$$
\int_{|x| \leqslant r+N(T-t)} H(u(x, t), K) d x \leqslant \int_{|x| \leqslant r+N T} H(u(x, 0), K) d x
$$

dove $H(u, k) \stackrel{\text { def }}{=} \bar{I}(u)-(j(k),(u-k))-\bar{I}(k)$ essenso $k$ un arbitrario vettore costante $d i$ $R^{n}, r>0, N=|E| \max _{|u|<\mid l}\left|f_{u}\right|$.

Poichè ogni soluzione $u$ deve verificare la disuguaglianza (28) qualunque sia $I \in D$ noi considereremo il caso in cui in $(28), I=H(u, k)$.

Allora ricordando che in questo caso $F_{u_{i}}=-E_{h j}\left(f_{h}(u)-f_{h}\left(k_{i}\right)\right)\left(f_{j}(u)-f_{j}\left(k_{i}\right)\right)_{u_{i}}$ e ponendo:

$$
G(u, k)=-\frac{1}{3} E_{h}\left(f_{h}(u)-f_{h}(k)\right)\left(f_{j}(u)-f_{j}\left(l_{i}\right)\right)
$$

si ha da (28):

$$
\int_{Q}\left\{H\left(u, l_{i}\right) \varphi_{\iota}+G\left(u, l_{i}\right) \varphi_{x}\right\} d x d t \geqslant 0
$$

da cui

$$
\int_{Q} H(u, k)\left\{\varphi_{i}+\frac{G(u, k)}{H(u, k)} \varphi_{x}\right\} d x d t \geqslant 0 .
$$

Poichè risulta per $|u|<M$

$$
\frac{G\left(u, f_{0}\right)}{H(u, k)} \leqslant|E| \max _{|u|<x \mid}\left|f_{x}\right|=N
$$

dove abbiamo indicato con $\left|f_{u}\right|$ il modulo della matrice di componenti $f_{i u_{j}}$.

Si ha così da (33)

$$
\int_{Q} H(u, k)\left\{p_{t}+N\left|\varphi_{x}\right|\right\} d x d t \geqslant 0
$$

Seguendo le medesime considerazioni adottate da $\mathbf{S}$. N. KRUžKov [22] nel caso di una singola equazione del tipo (8), definiamo $\alpha_{\gamma}(\sigma)=\int_{-\infty}^{\sigma} \delta_{\gamma}(\sigma) d \sigma$ e poniamo in $(34)$

$$
\varphi(x, t)=f(t) \chi^{\varepsilon}(x, t)
$$

dove $f \in C_{0}^{1}(0, T) \in \chi^{\varepsilon}(x, t)=1-\alpha_{\varepsilon}(|x|-N(T-t)-r+\varepsilon)$. 
Osserviamo inoltre che in virtù della definizione di $\chi^{\varepsilon}(x, t)$ abbiamo:

$$
f_{t} \chi^{\varepsilon}+f\left(\chi_{t}^{\varepsilon}+N\left|\chi_{x}^{\varepsilon}\right|\right)=f_{t} \chi^{\varepsilon}
$$

Pertanto da (34), ricordando che $H(u, k) \geqslant 0$, si ottiene:

$$
\int_{Q} f_{t} \chi^{\varepsilon} H(u, k) d x d t \geqslant 0
$$

Ora consideriamo in $(36) f(t)=\left[\alpha_{2}\left(t-t_{0}\right)-\alpha_{\gamma}\left(t-t_{1}\right)\right]$ dove i numeri $t_{0}, t_{1}$ appartengono, a meno di un insieme di misura nulla (vedi [22]), all'intervallo $\left(0, T_{0}\right)$, essendo $t_{0}<t_{1}$ e $T_{0}<T$. In tale caso (36) assume la forma:

$$
\int_{0}^{T}\left\{\delta_{\gamma}\left(t-t_{0}\right)-\delta_{\gamma}\left(t-t_{1}\right)\right\} \chi^{\varepsilon}(x, t) H(u, k) d x d t \geqslant 0
$$

per $\varepsilon \rightarrow 0$ si trova:

$$
\int_{0}^{r}\left\{\delta_{\gamma}\left(t-t_{0}\right)-\delta_{\gamma}\left(t-t_{1}\right)\right\} \int_{|冈| \leqslant x+N\left(T-t_{0}\right\}} H\left(u, t_{0}\right) d x d t \geqslant 0 .
$$

Infine per $\gamma \rightarrow 0$ otteniamo per quasi tutti i $t_{0}, t_{1} \in[0, T]$ la disnguaglianza:

$$
\int_{|x| \leqslant r+x\left(x-t_{1}\right)} H\left(u\left(x, t_{1}\right), k\right) d x \leqslant \int_{|x| \leqslant r+\Delta\left(x-t_{0}\right)} H\left(u\left(x, t_{0}\right), k\right) d x
$$

da cui per $t_{0} \rightarrow 0$ si ha $(31)$. Poichè $H(u, k)$ rappresenta l'energia interna per unità di massa a entropia costante, la disuguaglianza (31) traduce la legge di dissipazione di tale energia.

Se notiamo inoltre che la funzione $H(u, k)$ oltre ad annullarsi assume il suo minimo per $u=k$, possiamo concludere col formulare il seguente corollario del Teorema 1, valido per ogni arbitrario vettore costante $k$ :

Corollario. - Il problema di Cauchy (5), (2) con condizioni iniziali:

$$
\left.u\right|_{t=0}=k,
$$

ammette nella classe delle funzioni limitate e misurabili come unica soluzione generalizzata la funzione

$$
u(x, t) \equiv k
$$


MAURo FABRIZIo: Soluzioni generalizzate e disuguaglianze variazionali, ece.

5. - Comunque risulta tuttora problematica la formulazione di un teorema di dipendenza continua dai dati e quindi di unicità nel campo delle soluzioni generalizzate del problema di Cauchy (5), (2).

Tale impossibilità è probabilmente dovuta ad una ancora incompleta impostazione fisica.

Partendo da tale osservazione ho pensato ragionevole la considerazione secondo cui:

a) fra le eventuali soluzioni generalizzate del problema di Cauchy (5), (2) la soluzione che deserive correttamente il fenomeno risulta quella in corrispondenza della quale l'energia interna relativa ad ogni generica particella è minima.

In termini più precisi il principio a) è esprimibile nel seguente modo:

b) La soluzione generalizzata del problema di Cauchy (5), (2) che descrive il fenomeno in modo corretto risulta tale che per tutti $i t \in(0, T)$ :

$$
\int_{|x| \leqslant r+N(T-t)} H(u(x, t), k) d x=\min _{v \in \mathcal{S}} \int_{|x| \leqslant r+N \mid T-t)} H(v(x, t), k) d x
$$

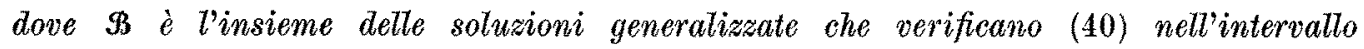
$(0, t-\delta)$ essendo $\delta>\delta_{0} \in(0, t / 2)$.

ì così possibile stabilire il seguente teorema:

TEOREMa 2. - Il problema di Cauchy(5), (2) ammette una unica soluzione generalizzata verificante la condizione $b$ ).

Infatti tutte le soluzioni del medesimo problema di Cauchy (quindi con le stesse condizioni iniziali) in conseguenza della condizione $b$ ) risultano tali che per ogni coppia di soluzioni $u_{1}, u_{2}$ si ha per tutti i $t \in(0, T)$ :

$$
\int_{|x| \leqslant r+N(x-t)} H\left(u_{1}, 7\right) d x=\int_{|x| \leqslant r+N(T-t)} H\left(u_{2}, k\right) d x
$$

ciò̀

$$
\int_{|x| \leqslant r+N(r-t)}\left\{\bar{I}\left(u_{1}\right)-\bar{I}\left(u_{2}\right)-\left(f(k), u_{1}-u_{2}\right)\right\} d x=0
$$

tale identità, dovendo risultare verificate per tutti i vettori costanti $k$ e quindi anche per quel valore $\bar{k}$ che minimizza $\bar{I}(u)$ e annulla $f(x)$, comporta:

$$
\vec{I}\left(u_{1}\right) \equiv \bar{I}\left(u_{2}\right) .
$$

Ora poichè $f(k)$ non può risultare per ogni $k$ sempre ortogonale ad $\left(u_{1}-u_{2}\right)$ abbiamo:

$$
u_{1} \equiv u_{2} .
$$




\section{BIBLIOGRAFIA}

[1] I. M. Gel'fand, Amer. Math. Soc. Transl., (2), 29 (1963), pp. $295-381$.

[2] B. L. Rozhonstvenskrr, Russian Math. Serveys, 15 (1960), pp. 53-111.

[3] J. Glimm - P. D. LaX, Proc. of Symp. in Pure Math., XVI (1970), pp. 233.236.

[4] O. A. Oxeinik, Amer. Math. Soc. Transl., (2), 26 (1963), pp. 95-172.

[5] J. Leray - Y. OHYA, Math. Ann., 170 (1967), pp. 167-205.

[6] K. O. Friedrichs, Comm. Pure Appl. Math., 7 (1954), pp. 345-393.

[7] S. K. Godunov, Russian Math. Serveys, 17 (1962), pp. 145-156.

[8] P. D. Lax, Comm. Pure Appl. Math., 7 (1954), pp. 159.193.

[9] E. Hopf, Comm. Pure Appl. Math., 3 (1950), pp. $201-230$.

[10] V. E. D'YaChenko, Math. USSR Sb., 2 (1961), pp. 7-9.

[11] N. D. VVedenskaya, Math. USSR Sb., 2 (1961), pp. 89.91.

[12] P. D. Lax, Non Linear Problems, The Univ. of Wisconsin, Madison, 1963, pp. 3.12

[13] A. E. Hurd, Pacif. Journal of Math., 29 (1969), pp. 555-559.

[14] M. Fabrizio, Atti Acc. Naz. dei Lincei, nota I, II, XLIX, fase. 3-4, 5, 1970.

[15] E. Hopx, Journ. of Math. and Mech., 19 (1969), pp. 483-487.

[16] S. N. KRUžKov, Soviet Math. Dokl., 10 (1969), pp. 785-788.

[17] A. I. VOL'PERT, Math. USSR Sb., 2 (1967), pp. 225-267.

[18] O. A. Orfinrk, Amer. Math. Soc. Transl., (2), 33 (1963), pp. 277-283.

[19] C. Truesdell - R. A. Toupin, Eneyclopedia of Physics, Vol. 1II/1, ed. S. Fü̈GGe, Pringer-Verlag, Berlin, Heidelberg, New York, 1960.

[20] C. Trunsdela - W. Nold, Eneyclopedia of Physics, Vol. III/3, ed. S. FlüGGe, PringerVerlag, Berlin, Heidelberg, New York, 1965.

[21] O. A. Ladyzenskaia - V. A. Solonnikov - N. N. Urai 'oeva, Transl. of Math. Monog. Vol. 23, 1968.

[22] S. N. KRUŽKov, Math. USSR Sb., 10 (1970), pp. 217.243. 